\title{
Die Nachtblindheit und ihre Behandlung in der griechisch- römischen Medizin
}

\author{
Ein Beitrag zur Geschichte der Mangelkrankheiten \\ von Nikolaus MaNi, Basel
}

Julius Hinschberg hat im ersten Bande seiner Geschichte der Augenheilkunde $^{13}$ in erschöpfender Weise die Belege aus der antiken medizinischen Literatur, in denen von der Nachtblindheit die Rede ist, angegeben und kritisch interpretiert. Da nun neuerdings Ärzte und Physiologen, wie Abderhalden ${ }^{1}$, Pillat ${ }^{23}$, Lindquist ${ }^{18}$, auf die Bedeutung der von den antiken Ärzten empfohlenen Behandlung der Nachtblindheit mit Leberzufuhr hingewiesen haben - ein Umstand, dem Hirschberg keine Beachtung schenkte -, soll hier versucht werden, die Berichte der alten Ärzte über die Nachtblindheit neu zu sichten und in den Rahmen der Geschichte der Mangelkrankheiten hineinzustellen.

Eine klare und unmißverständliche Definition der Nachtblindheit finden wir erst im post-hippokratischen Schrifttum. CELsus schreibt in seinem Werk Über die Medizin (VI, 6, 38. ${ }^{5}$ : «Praeter haec inbecillitas oculorum est, ex qua quidem interdiu satis, noctu nihil cernunt.» Er spricht hier von Augenpatienten, die tagsüber genügend sehen, in der Nacht aber nichts zu erkennen vermögen. GALEN ${ }^{6}$ erklärt in seinem Hippokrates-Glossarium,

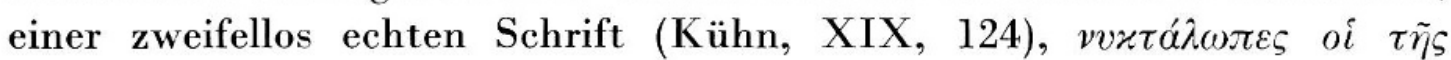

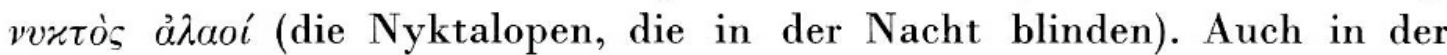
echten galenischen Schrift über die Methode der Heilkunst (KüHN, X, 84) versteht GaLEN unter den «Nyktalopen» Kranke, die in der Nacht nichts

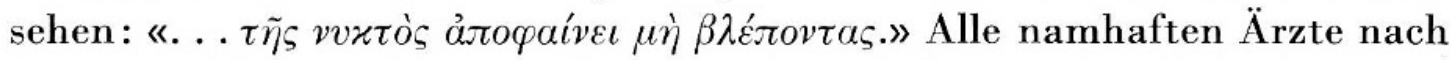
GALEN schließen sich ausnahmslos seiner Definition der Nyktalopie an. Oribasius ${ }^{21}$ definiert die Nyktalopie mit folgenden Worten (Synopsis

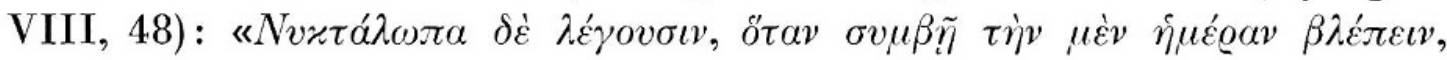

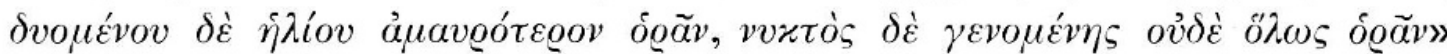
(Von Nyktalopie spricht man dann, wenn die Sehkraft tagsüber gut ist, nach Sonnenuntergang abnimmt und man in der Nacht überhaupt nichts mehr zu unterscheiden vermag). Aёtios aus Amida ${ }^{3}$ und Paulus von Aegina $^{22}$ folgen beinahe wörtlich dieser von Oribasıus gegebenen Bestimmung der Nyktalopie. Unter den Termini «Nyktalops», «Nyktalopiasis»

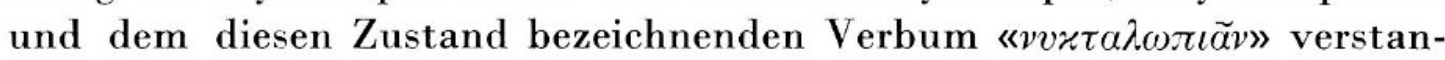
den Galen, Oribasius, Aëtrus und Paulus von Aegina Nachtblindheit. 
Auch die von Celsus beschriebene Sehschwäche (inbecillitas oculorum) weist die gleichen Symptome auf.

Für die Behandlung der Nachtblindheit verordnen all diese genannten Ärzte das Essen gebratener oder gekochter Leber von Ziegen und Ziegenböcken. Neben der internen Ziegenlebertherapie empfahlen sie auch eine lokale Behandlung: Der aus der bratenden Leber fließende Saft soll auf die Augen gestrichen werden. GaLEN sagt in der Schrift De compositione medicamentorum secundum locos (KüHN, XII, 802/803): «Von der Leber gib (den Nachtblinden) fortwährend zu essen, tue dies, dann wird diesen Kranken schnell geholfen werden.» Ä̈тıUs erprobte die Lebertherapie an Tag- und Nachtblinden und machte dabei die Erfahrung, daß mit dieser Behandlung die Nachtblindheit besser beeinflußt wurde als die seltener vorkommende Tagblindheit ${ }^{3}$ (S. 120). In der im Corpus Hippocraticum enthaltenen Schrift Über das Sehen wird für die Behandlung des «Nyktalops» das ein- bis zweimalige Einnehmen einer möglichst großen Menge roher Rindsleber verordnet (Lit. IX, 158).

Nach Abderhalden-Mouriquand sind in $8 \mathrm{~g}$ der Leber von Wiederkäuern etwa 1000 I.E. Vitamin A enthalten. Die von den alten Ärzten festgestellte gute Wirkung der Lebertherapie bei Nachtblindheit ist auf den reichen Gehalt der Leber an Vitamin A zurückzuführen. Die häufige Erwähnung der Hemeralopie in der alten medizinischen Literatur und die Berichte über ihre erfolgreiche Behandlung durch Leberzufuhr weisen darauf hin, daß die Mangelhemeralopie - die als eines der ersten Zeichen der A-Avitaminose auftritt - in diesen Zeiten keine seltene Erscheinung war. Die ungenügende Ernährung der armen Bevölkerungsschichten und der Sklaven, die Ernährungsschwierigkeiten in Kriegszeiten und bei Mißernten führten sehr wahrscheinlich zu einem gehäuften Auftreten der Nachtblindheit. Die Sichtung neuerer Berichte über das Vorkommen der Nachtblindheit im 19. und 20. Jahrhundert ${ }^{17,23,25}$ läßt diese Vermutung an Wahrscheinlichkeit gewinnen. GRAEFE beobachtete 1858 an den Züchtlingen einer Strafanstalt in Halle mehrere Fälle von Hemeralopie ${ }^{10}$, ВІтот beschrieb 1863 die nach ihm benannten, zusammen mit Nachtblindheit auftretenden Flecken an der Augenbindehaut ${ }^{4}$ bei Kindern aus einem Waisenhaus in Bordeau, und VILLEMIN berichtete über eine «kleine Epidemie von Nachtblindheit» bei Angehörigen eines in Straßburg kasernierten Bataillons ${ }^{28}$. GouvEA beschrieb 1883 zahlreiche Fälle von Nachtblinheit bei brasilianischen Negersklaven ${ }^{8}$, und aus Rußland meldeten verschiedene Autoren das epidemische Auftreten von Nachtblindheit während der Osterfastenzeit ${ }^{15,24,26,30}$ und bei Hungersnöten. In Rußland, wo die Nachtblindheit epidemisch auftrat, war das klassische Heilmittel der griechischen Medizin, die Leber, als Volksheilmittel gegen die Hemeralopie wohlbekannt ${ }^{11,15}$ und verschiedene Ärzte konnten die gute Wirkung desselben bestätigen ${ }^{15,24}$. Auch in China, «dem klassischen Lande der Hemeralopie», stellten Pillat ${ }^{23}$, Hou ${ }^{14}$ und Gow ${ }^{9}$ die große Häufigkeit der Nachtblindheit fest. Die Ursache dieser weiten Verbreitung der Hemeralopie in China ist darauf zurückzuführen, daß die ärmeren Bevölkerungsschichten nur in ungenügender Weise ihren Vitamin-A-Bedarf mit Milch, Milchprodukten, Gemüse und Fleisch decken 
können. Auch bei den russischen Fasten wurde eine einseitige Zerealienkost beobachtet, wobei der Genuß von Fleisch und tierischen Produkten, wie Milch, Butter und Eier, verboten war $^{32}$.

Ähnlich ungenügende Ernährungsverhältnisse dürfen wir wohl auch für das Altertum annehmen. Es ist wahrscheinlich kein Zufall, daß in den hippokratischen Schriften über die Volkskrankheiten das Vorkommen von «Nyktalopie» mehrmals erwähnt wird. Im vierten Buche der Epidemien (Lit. V, 193) wird bei «nyktalopen» Kindern fiebriger Husten mit Ohrenentzündung beschrieben.

Pillat hat nun an Kindern und Erwachsenen Bronchitiden beschrieben, die einzig durch Vitamin-A-Zufuhr schlagartig gebessert wurden ${ }^{23}$. Die zusammen mit fiebrigem Husten auftretende «Nyktalopie» an der eben erwähnten Stelle der Volkskrankheiten lassen die Hypothese zu, daß es sich hier möglicherweise um eine A-Avitaminose handelte, die natürlich uach mit anderen Avitaminosen gekoppelt gewesen sein kann.

Im 6. Buche der Epidemien (Lit. V, 330-335) wird eine Hustenepidemie beschrieben, bei der neben «Nyktalopie» fieberhafte Krankheiten der Atmungswege beobachtet wurden.

Einer einigermaßen befriedigenden Interpretation der eben angeführten Stellen aus den «Volkskrankheiten» stellt sich folgende Schwierigkeit entgegen: Es wird hier nirgends gesagt, daß unter den «Nyktalopen» Nachtblinde verstanden wurden. Anderseits bezeichnet der Autor des zweiten Buches der Vorhersagungen (Lit. IX, 64) die Nyktalopen als Tagblinde, d. h. «in der Nacht Sehende» (oi $\delta \dot{\varepsilon} \tau \tilde{\eta} \varsigma v v \varkappa \tau o ̀ \varsigma ~ \delta \varrho \tilde{\omega} v \tau \varepsilon \varsigma)$. Diese Definition wurde von Hirschberg ${ }^{13}$, Fuchs und Kapferer ${ }^{12}$ auch auf die in den hippokratischen «Epidemien» beschriebenen Nyktalopie-Fälle übertragen. Dagegen sollen folgende Einwände erhoben werden:

1. Man kann nicht ohne weiteres die Definition der Nyktalopie in den Vorhersagungen auch auf die in den Epidemien beschriebenen Nyktalopien übertragen.

2. GALEN zumindestens erklärte den im hippokratischen Schrifttum vorkommenden Terminus «Nyktalops» als Nachtblindheit (KüHN, XIX, 124).

3. In einer Handschrift, welche die erwähnte Stelle in den Vorhersagungen

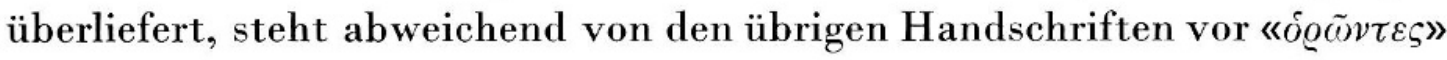

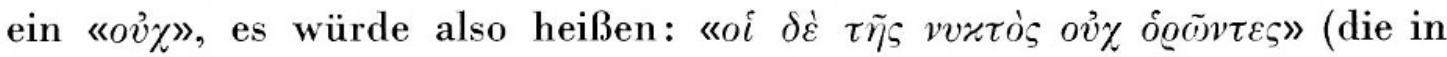
der Nacht nicht Sehenden) (Lit. IX, 65, Anm. 9).

4. In der erwähnten Stelle der Vorhersagungen wird die «Nyktalopie» als eine vorübergehende Krankheit geschildert. Sie befalle vor allem Kinder und Jugendliche, nicht aber Frauen und Jungfrauen, bei denen die Regeln 
bereits erschienen seien. CELsus, der sich bei der Beschreibung der Nachtblindheit offensichtlich auf diese Stelle der Vorhersagungen bezieht, stellt das gleiche fest: Die Nachtblindheit befällt die Frauen mit normalen Regeln nicht.

Nun weist Stepp darauf hin, daß die geschlechtsreife Frau für das Vitamin A ein starkes Speicherungsvermögen besitze. Birnbacher (zit. nach STEPP ${ }^{25}$, S. 81-82) stellte fest, daß Frauen im zweiten und dritten Jahrzehnt seltener an Hemeralopie erkrankten als Männer der gleichen Altersstufe. Auch Kinder sind besonders empfindlich gegen Vitamin-A-Mangel ${ }^{25}$.

5. Littré und Hirschberg glaubten, daß es sich bei den «Nyktalopen» der Vorhersagungen um Kinder mit «Hornhautentzündung und Lichtscheu» gehandelt habe. Nun ist aber Lichtscheu zusammen mit Hemeralopie ein Frühsymptom des Vitamin-A-Mangels.

6. Das epidemische Auftreten der nyktalopischen Sehstörung, wie es im vierten und sechsten Buche der Epidemien beschrieben wird, lassen eher an Nacht- als an Tagblindheit denken. Dafür spricht auch das gehäufte Vorkommen der «Nyktalopie» in den erwähnten Stellen der Epidemien bei Kindern.

Anderseits ist zu sagen, daß im post-hippokratischen Schrifttum eine doppelte Bedeutung des Wortes «Nyktalopie» bekannt war ${ }^{13}$. Man könnte auch vermuten, daß die Photophobie der Nachtblinden oder gar die zusammengekniffenen Augenlider der keratomalazischen Kinder als «Tagblindheit» imponierten.

Unter den im Corpus Hippocraticum gesammelten Schriften wird einzig in der kleinen Schrift Über das Sehen von der Behandlung der «Nyktalopie» gesprochen. Die Therapie besteht in einer ein- bis zweimaligen Zufuhr einer möglichst großen Menge roher Leber (Lit. IX, 158).

Mit einer ähnlichen Behandlung, nämlich dem ein- bis zweimaligen Essen von je $150 \mathrm{~g}$ Leber, sah $\mathrm{Z}_{\mathrm{AK}}{ }^{32}$ im Ersten Weltkrieg während seiner Kriegsgefangenschaft in Rußland ein rasches Abklingen der Hemeralopie. 1901 berichtete Trantas, der am hellenischen Hospital in Konstantinopel als Augenarzt wirkte, von einer schlagartigen Besserung der Nachtblinheit bei 40 Waisenhauskindern nach der drei- oder viermaligen Gabe von $200 \mathrm{~g}$ gekochter Hammelleber. Ähnlich gute Resultate mag auch der Autor der Schrift Über das Sehen gehabt haben. Ä̈тrus und GaLeN bezeugen auf jeden Fall die gute Wirkung der Lebertherapie bei Nachtblinden.

In neuerer Zeit ${ }^{23}$ wurde vom «Frühlingsgipfel» der Mangelhemeralopie gesprochen. Der im 14. Jahrhundert lebende byzantinische Arzt Johannes Aктuarius berichtet von den

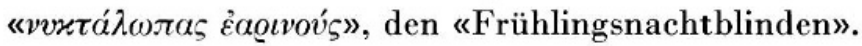

Zusammenfassend soll festgestellt werden: In der Antike wird im allgemeinen unter «Nyktalopie» Nachtblindheit verstanden. Diese Sehstörung 
wird im medizinischen Schrifttum der Antike und der byzantinischen Zeit häufig erwähnt. Die Nachtblindheit war eine den Ärzten wohlbekannte Erscheinung. Die Häufigkeit der Nachtblindheit und die gute Wirkung, welche die Lebertherapie nach den Berichten der alten Ärzte auf dieses Leiden ausübte, erlaubt die Annahme, daß es sich bei dem gehäuften Auftreten der Nachtblindheit nicht um die hereditäre oder die bei Retinitis pigmentosa und anderen organischen Augenaffektionen vorkommende Nachtblindheit handelte, sondern um die bei Vitamin-A-Mangel erscheinende Hemeralopie. Bei der epidemischen «Nyktalopie», die in den Volkskrankheiten des hippokratischen Schrifttums beschrieben wird, dürfen wir ebenfalls mit einiger Wahrscheinlichkeit annehmen, daß damit Nachtblindheit gemeint ist. Die Nachtblindheit, eine sehr eindrückliche und auffallende Erscheinung, ist wohl eines der historisch am besten faßbaren avitaminotischen Symptome.

\section{Literatur}

${ }^{1}$ E. Abderhalden, G. Mouriquand, Vitamine und Vitamintherapie, Bern 1948; ${ }^{2}$ Actuarii Johannis opera, Lugduni 1556; ${ }^{3}$ Aetros, J.Hirschberg, Die Augenheilkunde des Aëtius aus Amida, Leipzig 1899; ${ }^{4}$ Вітот, Mémoire sur une lésion conjonctivale non encore décrite coüncidant avec l'héméralopie, Gaz. Hebdom. de Méd. et de Chir. X, 1863 (S. 284-288); ${ }^{5}$ Aulus Connelrus Celsus, Corp. Med. Latin., Vol. I, Lips. Berol. 1915; ${ }^{6}$ Galen, C. G. KüHn, Op. Med. Graec., Vol. I-XX, Lips. 1821-1830; 7 H. K. Goldberg et al,. Necrosis of the Cornea due to Vitamin A Deficiency, Arch. of Opth. 25, 1941 (S. 122-127); ${ }^{8}$ H. DE GouvêA, Beiträge zur Kenntnis der Hemeralopie und Xerophthalmie aus Ernährungsstörungen, Arch. f. Ophth. 29., Abt. 1, 1883 (S. 167-200); ${ }^{9}$ W. H. Gow, Some Clinical Observations on Cases of Keratomalacia in Manchuria, Chinese Medical Journal 48, 1934. (S. 885-889); ${ }^{10}$ A.Graefe, Beiträge zum Wesen der Hemeralopie, Arch. f. Ophth. 5, 1859 (S. 112-127); 11 Ant. Alf. Henrici, Weitere Studien über Volksheilmittel verschiedener in Rußland lebender Völkerschaften, in Histor. Stud.pharmakol. Institut Univ. Dorpat, IV, hgg. v. R. Kobert, Halle a. d.S. 1894; ${ }^{12}$ Hippokrates, E. LitTré, Euvres compl. d'Hippocrate, Vol. I-X, Paris 1839-1861, R. Fuchs, Hippokrates' sämtliche Werke, München 1895-1900, R. Kapferer, Die Werke des Hippokrates, Stuttgart-Leipzig 1934; ${ }^{13}$ J. HirschBERG, Geschichte der Augenheilkunde im Alterthum, Leipzig 1899; 14 H. CH. Hou, The Relation of Vitamin A to Health and Disease, Chinese Medical Journal 50, 2. Abt. 1936 (S. 1481-1492); ${ }^{15}$ Tн. Kubli, Zur Lehre von der epidemischen Hemeralopie, Arch. f. Augenheilkd. 17, 1887 (S. 409-411); ${ }^{16}$ Th. Leber, Über die Xerosis der Bindehaut und die infantile Hornhautverschwärung, Arch. f. Ophth. 29, Abt. III, 1883; 17 Th. Leber in GraefeSAEmisch, Handb. d. gesamt. Augenheilkd., Bd. V, Leipzig 1877 (S. 994-1004); 18 T. LINDQUIST, Studien über das Vitamin A beim Menschen, Acta Med. Scandinav., Suppl. XCVII, 1938; ${ }^{19}$ K. Lohmann, Einfluß von Licht und Dunkelheit auf den Stoffwechsel, Dtsch. Med. Wschr. 66, 1, 1940 (S. 569-57I); ${ }^{20}$ OEFELE, Leberpräparate in der Ophthalmologie einst und jetzt, Ophthalm. Klinik, $V, 1901$ (S. 101); ${ }^{21}$ Oribasius, Daremberg-Bussemaker, CEuvres 
d'Oribase, Bd. V, Paris 1873; ${ }^{22}$ Paulus Aegineta, Corp. Med. Graec., ed. J. L. Heiberg, Lips. Berol 1921, Bd. IX, 1; ${ }^{23}$ A. Pillat, Mangel an Vitamin A, in Stepp, Ernährungslehre, Berlin 1939 (S. 283-330); ${ }^{24}$ N. SснтsсhеротJEW, Ein Beitrag zur Lehre von der epidemischen Hemeralopie, Arch. f. Augenheilkd. 32, 1896 (S. 194-197); ${ }^{25}$ W. STEPP, J. Kühnau, H. Schroeder, Die Vitamine und ihre klinische Anwendung, Bd. I, Stuttgart 1952; ${ }^{26}$ J. Thalberg, Zur Casuistik der durch Inanitionszustände bedingten Hornhautgangrän, Arch. f. Augenheilkd. 12, 1883 (S. 315-332); 27 Trantas, Traitement de l'héméralopie essentielle par l'ingestion du foie bouilli ou cuit, Arch. d'Ophth. 21, 1901 (S. 723-726); ${ }^{28}$ J. A. Villemin, De l'altération épithéliale de la conjonctive oculaire dans l'héméralopie, Gaz. Hebdom. de Méd. et de Chir. X, 1863 (S. 332-335); ${ }^{29}$ A. Villaret, Handwörterb. d. ges. Mediz., I. Bd., 1888 (S. 829); ${ }^{30}$ O. WALter, Ein Beitrag zur Lehre von der epidemischen Nachtblindheit, Arch. f. Augenheilkd. 27, 1893 (S. 71-99); ${ }^{31}$ E. Wieland, Zur Ätiologie, Pathogenese und moderner Therapie der A-Avitaminose (Keratomalacie) beim Säugling, Mailand 1934; ${ }^{32}$ E. ZAK, Wien. Med. Wschr. 70, 1920 (S. 600-601). 